\title{
EXTENSIONS OF $G$-POSETS AND QUILLEN'S COMPLEX
}

\author{
YOAV SEGEV and PETER WEBB
}

(Received 8 September 1993)

Communicated by L. G. Kovács

\begin{abstract}
We develop techniques to compute the homology of Quillen's complex of elementary abelian $p$-subgroups of a finite group in the case where the group has a normal subgroup of order divisible by $p$. The main result is a long exact sequence relating the homologies of these complexes for the whole group, the normal subgroup, and certain centralizer subgroups. The proof takes place at the level of partially-ordered sets. Notions of suspension and wedge product are considered in this context, which are analogous to the corresponding notions for topological spaces. We conclude with a formula for the generalized Steinberg module of a group with a normal subgroup, and give some examples.
\end{abstract}

1991 Mathematics subject classification (Amer. Math. Soc.): primary 20D30; secondary 05E25, 06A09, $20 \mathrm{C} 20,51 \mathrm{E} 25$.

\section{Introduction}

Let $G$ be a finite group and $p$ a prime. Let $\mathscr{A}_{p}(G)$ be the Quillen complex of $G$ at the prime $p . \mathscr{A}_{p}(G)$ is the order complex of the poset (= partially ordered set) of all non-trivial elementary abelian $p$-subgroups of $G$ (see Section 1 ).

Let $N$ be a normal subgroup of $G$. We denote by $\mathscr{A}_{p}(G)_{N}$ the poset obtained by adding to $\mathscr{A}_{p}(G)$ an additional element, say 0 , so that $0<A$ for all $A \in \mathscr{A}_{p}(G)$ with $A \cap N \neq 1$. Our main result is the following.

MAIN THEOREM. Let $G$ be a finite group and $p$ a prime. Suppose that $N$ is a normal subgroup of $G$ such that $p$ divides $|N|$. Further except in (1) assume that if $A$

(C) 1994 Australian Mathematical Society 0263-6115/94 \$A2.00+0.00

The second author was partially supported by the NSF. 
is an elementary abelian p-subgroup of $G$ with $A \cap N=1$ then $A$ is cyclic, and let $\mathscr{H}=\left\{A \in \mathscr{A}_{p}(G) \mid A \cap N=1\right\}$.

(1) There exists a long exact sequence of $\mathbb{Z} G$-modules

$$
\cdots \rightarrow \tilde{H}_{n}\left(\mathscr{A}_{p}(N)\right) \stackrel{\iota_{*}}{\longrightarrow} \tilde{H}_{n}\left(\mathscr{A}_{p}(G)\right) \stackrel{\kappa_{*}}{\longrightarrow} \tilde{H}_{n}\left(\mathscr{A}_{p}(G)_{N}\right) \stackrel{s}{\longrightarrow} \tilde{H}_{n-1}\left(\mathscr{A}_{p}(N)\right) \rightarrow \cdots
$$

where $\iota_{*}$ and $\kappa_{*}$ are induced by the obvious inclusion maps $\iota$ and $\kappa$.

(2) There is a G-homotopy equivalence

$$
\mathscr{A}_{p}(G)_{N} \simeq_{G} \bigvee_{A \in \mathscr{M}} \Sigma \mathscr{A}_{p}\left(C_{N}(A)\right)
$$

where $\bigvee$ denotes $a$ wedge product and $\Sigma$ denotes suspension.

(3) For $n \geq 0$

$$
\begin{aligned}
\tilde{H}_{n}\left(\mathscr{A}_{p}(G)_{N}\right) & \cong \bigoplus_{A \in \mathcal{A}} \tilde{H}_{n-1}\left(\mathscr{A}_{p}\left(C_{N}(A)\right)\right) \\
& \cong \bigoplus_{\substack{A \in \mathcal{H} \\
\text { up to conjugacy }}} \tilde{H}_{n-1}\left(\mathscr{A}_{p}\left(C_{N}(A)\right)\right) \uparrow_{N_{G}(A)}^{G}
\end{aligned}
$$

as $\mathbb{Z} G$-modules.

(4) With respect to the first isomorphism of (3) the map s of (1) is given by

$$
s=\bigoplus_{A \in \mathscr{M}}\left(\iota_{A}\right)_{*}
$$

where $\left(\iota_{A}\right)_{*}$ is the map on homology induced by the inclusion $\iota_{A}: \mathscr{A}_{p}\left(C_{N}(A)\right) \rightarrow$ $\mathscr{A}_{p}(N)$.

We will prove the Main Theorem in the abstract setting of a poset $P$ having a subposet $Q$ satisfying the two conditions that the elements of $Q$ form an ideal in the opposite poset $P^{\text {op }}$, and for each $p \in P$ there exists $q \in Q$ with $q \geq p$. In this situation we will call $P$ an extension of $Q$, and in the context of the Main Theorem we will take $P=\mathscr{A}_{p}(G)$ and $Q=\left\{A \in \mathscr{A}_{p}(G) \mid A \cap N \neq 1\right\}$. We give our sharpest results when $P-Q$ consists of minimal elements of $P$, and in this generality Theorem 2.5 gives an inductive set-up for dealing with the homology of a poset.

After proving the Main Theorem in Section 3, we consider the generalized Steinberg module $S t_{p}(G)$ in the situation that $G$ has a normal subgroup $N$ of order divisible by $p$. Because the computation of $S t_{p}(G)$ does not require such detailed information as the homology of $\mathscr{A}_{p}(G)$, we are able to remove the condition that $A \in \mathscr{A}_{p}(G)$, $A \cap N=1$ implies $A$ is cyclic which was present in part of the Main Theorem, and obtain a result in generality. This result is Theorem 4.2. We conclude in Section 5 with two worked examples. 


\section{The Mayer-Vietoris sequence for an extension of posets}

We first describe a poset construction which gives rise to a Mayer-Vietoris sequence. Let $P$ be a poset. The order complex of $P$ is the simplicial complex $\Delta P$ whose simplices are the finite chains of elements of $P$. We may occasionally omit the symbol $\Delta$, especially when considering the homology $H_{n}(P)$, by which we mean $H_{n}(\Delta P)$. Given a subposet $Q \leq P$ and $p \in P$ we denote by $Q_{\geq p}$ the subposet of $P$ with elements $\{q \in Q \mid q \geq p\}$. We define $Q_{>p}, Q_{<p}$ etc. in a similar way. An ideal of $P$ is a subposet $\emptyset \neq I \leq P$ such that if $i \in I$ and $p \leq i$ then $p \in I$.

Let $Q \leq P$ be a subposet. We say that $P$ is an extension of $Q$ if $Q^{\text {op }}$ is an ideal of the opposite poset $P^{\text {op }}$, and for all $p \in P, Q_{\geq p} \neq \emptyset$. We will further say that $P$ is an extension of $Q$ by minimal elements if $P$ is an extension of $Q$ and for all $p \in P$, either $p \in Q$ or $p$ is a minimal element of $P$. If $P$ is an extension of $Q$ we denote by $P_{Q}$ the following poset. The elements of $P_{Q}$ are $P$ together with one additional element $0_{Q}$. The order relation in $P_{Q}$ is the following. Given $x, y \in P_{Q}, x<y$ if and only if either $x, y \in P$ and $x<y$ in $P$, or $x=0_{Q}$ and $y \in Q$. We denote by $Q_{Q} \leq P_{Q}$ the similarly constructed poset with elements $Q \cup\left\{0_{Q}\right\}$. If $P$ is a $G$-poset and $Q$ is $G$-invariant, then $P_{Q}$ becomes a $G$-poset by letting $G$ fix $0_{Q}$.

Turning to our conventions for homology, we first mention that unless otherwise specified all homology groups are taken with coefficients in $\mathbb{Z}$. Let $K$ be a simplicial complex. We denote by $C_{n}(K)$ the simplicial chain group of $K$ at dimension $n$ (with coefficients in $\mathbb{Z}$ ), $n \geq 0 . \quad \tilde{C}_{n}(K)$ denotes the augmented simplicial chain group of $K$ at dimension $n, n \geq-1$. So $\tilde{C}_{n}(K)=C_{n}(K)$ for all $n \geq 0$, and $\tilde{C}_{-1}(K)=\mathbb{Z}$. By $Z_{n}(K)$ respectively $B_{n}(K)$ respectively $H_{n}(K)$ we denote the group of $n$-cycles respectively $n$-boundaries respectively $n$-homology group. The notation $Z_{*}(K), H_{*}(K), \tilde{Z}_{n}(K), \tilde{H}_{n}(K), \tilde{H}_{*}(K)$ etc. is now clear. Given a cycle $z \in \tilde{Z}_{n}(K)$ we denote by $[z]=z+\tilde{B}_{n}(K)$ the corresponding element in $\tilde{H}_{n}(K)$.

PROPOSITION 1.1. Let $P$ be an extension of $Q$. Then

(1) $\Delta P_{Q}=\Delta P \cup \Delta Q_{Q}$ and $\Delta P \cap \Delta Q_{Q}=\Delta Q$.

(2) There is a long exact Mayer-Vietoris reduced homology sequence

$$
\cdots \rightarrow \tilde{H}_{n}(Q) \stackrel{\cdot}{\longrightarrow} \tilde{H}_{n}(P) \stackrel{\kappa_{*}}{\longrightarrow} \tilde{H}_{n}\left(P_{Q}\right) \stackrel{r}{\longrightarrow} \tilde{H}_{n-1}(Q) \rightarrow \cdots
$$

where $\iota_{*}, \kappa_{*}$ are the maps on homology induced by the obvious inclusion maps $\iota, \kappa$ and $r$ is given as follows. If $\alpha \in \tilde{C}_{n}(P)$ and $\beta \in \tilde{C}_{n}\left(Q_{Q}\right)$ are such that $\partial(\alpha+\beta)=0$, then $r([\alpha+\beta])=[\partial \alpha]$, where $\partial$ is the differential map of $P_{Q}$. In case $P$ is a $G$-poset and $Q$ is $G$-invariant, the Mayer-Vietoris sequence is a sequence of $\mathbb{Z} G$-modules.

PROOF. The Mayer-Vietoris sequence needs no proof. We only mention that as $Q_{Q}$ is contractible, $\tilde{H}_{*}\left(Q_{Q}\right)=0$. 


\section{The structure of $P_{Q}$}

The use of the Mayer-Vietoris sequence just described is considerably enhanced by the fact that we are able to give an explicit description of the space $\Delta P_{Q}$ in case $P$ is an extension of $Q$ by minimal elements, showing that it is homotopic to a wedge of suspensions of certain other posets. To define what we mean by this, we make definitions at the level of posets which copy well-known topological constructions.

First let $R$ be a poset. In this paper by the suspension of $R$ we mean a poset

$$
\Sigma R=R \cup\left\{0_{R}, 0_{R}^{\prime}\right\}
$$

where $0_{R}$ and $0_{R}^{\prime}$ are two new symbols, and where the order relation is as follows. For $x, y \in \Sigma R$ we define $x \leq y$ if and only if either $x=0_{R}$ and $y \neq 0_{R}^{\prime}$, or $x=0_{R}^{\prime}$ and $y \neq 0_{R}$, or $x, y \in R$ and $x \leq y$ in $R$. In case $R$ is a $G$-poset then $G$ acts on $\Sigma R$ by fixing $0_{R}$ and $0_{R}^{\prime}$.

To describe also the action of $G$ on the homology of $R$ and of $\Sigma R$ we introduce the following notation. Given an $(n-1)$-simplex $s=\left(r_{0}<r_{1}<\cdots<r_{n-1}\right)$ of $R$ and $r \in R$ with $r<r_{0}$, we denote $r * s=\left(r<r_{0}<r_{1}<\cdots<r_{n-1}\right)$. Let $n \geq 1$. Given a cycle $z \in \tilde{Z}_{n-1}(R)$, write $z=\sum_{i=1}^{m} n_{i} s_{i}$, with $s_{i}$ an $(n-1)$-simplex of $R$. We write $0_{R} * z=\sum_{i=1}^{m} n_{i}\left(0_{R} * s_{i}\right) \in \tilde{C}_{n}(\Sigma(R))$ and similarly we define $0_{R}^{\prime} * z \in \tilde{C}_{n}(\Sigma(R))$. We write $\Sigma(z)=0_{R} * z-0_{R}^{\prime} * z$.

PROPOSITION 2.1. Suppose that $R$ is a G-poset.

(1) There is a G-equivariant homeomorphism $\Delta(\Sigma R) \cong_{G} \Sigma \Delta(R)$.

(2) For $n \geq 1$, if $z \in \tilde{Z}_{n-1}(R)$ then $\partial\left(0_{R} * z\right)=\partial\left(0_{R}^{\prime} * z\right)=z$, and hence $\Sigma(z) \in \tilde{Z}_{n}(\Sigma R)$. Here $\partial$ is the differential map of $\Sigma(R)$.

(3) The map $\tilde{H}_{n-1}(R) \rightarrow \tilde{H}_{n}(\Sigma R)$ given by $[z] \rightarrow[\Sigma(z)]$ is an isomorphism of $\mathbb{Z} G$-modules.

PrOOF. In part (1) the action of $G$ on $\Sigma \Delta(R)$ fixes the suspension coordinate and acts on $\Delta(R)$ in the given way. The homeomorphism is immediate, since each of the subposets $R \cup\left\{0_{R}\right\}$ and $R \cup\left\{0_{R}^{\prime}\right\}$ is a cone on $R$ with vertex fixed by $G$. The description in parts (2) and (3) is well-known.

Continuing in this vein, let $\left\{R_{t} \mid t \in \mathscr{T}\right\}$ be a set of posets indexed by some set $\mathscr{T}$. By a wedge of suspensions of the $R_{t}$ we mean a poset

$$
X=\bigcup_{t \in \mathscr{T}}\left(R_{t} \times\{t\}\right) \cup \mathscr{T} \cup\{0\}
$$

which we will denote by $\bigvee_{t \in \mathscr{T}} \Sigma R_{t}$. We define a partial order on this set as follows. For $t \in \mathscr{T}$ define

$$
j_{t}: R_{t} \times\{t\} \rightarrow R_{t}
$$


by $j_{t}(r, t)=r$. If $x, y \in X$ we put $x<y$ if and only if one of the following holds:

(i) there exists $t \in \mathscr{T}$ such that $x, y \in R_{t} \times\{t\}$ and $j_{t}(x)<j_{t}(y)$,

(ii) $x=t \in \mathscr{T}$ and $y \in R_{t} \times\{t\}$,

(iii) $x=0$ and $y \notin \mathscr{T} \cup\{0\}$.

The point about using the set $R_{t} \times\{t\}$ in the above construction is that it guarantees that all these sets are disjoint as $t$ ranges through $\mathscr{T}$. However, when no confusion may arise we identify $R_{t}$ with $R_{t} \times\{t\}$ via $j_{t}$. We do this in the next result.

PROPOSITION 2.2. Let $\left\{R_{t} \mid t \in \mathscr{T}\right\}$ be posets. Then

(1) $\Delta\left(\bigvee_{t \in \mathscr{T}} \Sigma R_{t}\right) \cong \bigvee_{t \in \mathscr{T}} \Sigma \Delta\left(R_{t}\right)$, and

(2) for $n \geq 1$ the map

$$
\mu: \bigoplus_{t \in \mathscr{T}} \tilde{H}_{n-1}\left(R_{t}\right) \rightarrow \tilde{H}_{n}\left(\bigvee_{t \in \mathscr{T}} \Sigma R_{t}\right)
$$

defined by $\mu\left(\sum_{t \in \mathscr{T}}\left[z_{t}\right]\right)=\sum_{t \in \mathscr{T}}\left[t * z_{t}-0 * z_{t}\right]$ is an isomorphism, where for all $t \in \mathscr{T}, z_{t} \in \tilde{Z}_{n-1}\left(R_{t}\right)$.

PROOF. (1) is immediate from the definitions and (2) is well-known.

We will apply this construction in the situation where $P$ is an extension of $Q$ by minimal elements. We take the indexing set $\mathscr{T}$ to be the set $\mathscr{M}=P-Q$, which consists of minimal elements of $P$, and the posets $R_{t}$ are the $P_{>m}, m \in \mathscr{M}$. In this situation, if $P$ is a $G$-poset and $Q$ is $G$-invariant we may define a group action on the poset $\bigvee_{m \in \mathscr{M}} \Sigma P_{>m}$ by

$$
\begin{aligned}
g(p, m) & =(g p, g m), \quad \text { for }(p, m) \in P_{>m} \times\{m\}, \\
g m & =g m, \quad m \in \mathscr{M}, \\
g 0 & =0,
\end{aligned}
$$

and now the homology groups of this poset become $\mathbb{Z} G$-modules. There is also a group action on the simplicial complex

$$
\bigvee_{m \in \mathscr{M}} \Sigma \Delta\left(P_{>m}\right)
$$

in which if $x \in \Sigma \Delta\left(P_{>m}\right)$ and $g \in G$ then $g x \in \Sigma \Delta\left(P_{>g m}\right)$.

PROPOSITION 2.3. Suppose that $P$ is a G-poset which is an extension of $Q$ by minimal elements where $Q$ is $G$-invariant. Put $\mathscr{M}=P-Q$.

(1) There is a G-equivariant homeomorphism

$$
\Delta\left(\bigvee_{m \in \mathscr{M}} \Sigma P_{>m}\right) \cong{ }_{G} \bigvee_{m \in \mathscr{M}} \Sigma \Delta\left(P_{>m}\right)
$$


(2) For $n \geq 1$ the group $\bigoplus_{m \in \mathscr{M}} \tilde{H}_{n-1}\left(P_{>m}\right)$ acquires the structure of an induced $\mathbb{Z} G$-module

$$
\bigoplus_{m \in \mathscr{M}} \tilde{H}_{n-1}\left(P_{>m}\right) \cong \bigoplus_{m \in[G \backslash \mathscr{M}]} \tilde{H}_{n-1}\left(P_{>m}\right) \uparrow_{G_{m}}^{G}
$$

where $G_{m}$ is the stabilizer of $m$ in $G$. The mapping

$$
\mu: \bigoplus_{m \in \mathscr{M}} \tilde{H}_{n-1}\left(P_{>m}\right) \rightarrow \tilde{H}_{n}\left(\bigvee_{m \in \mathscr{K}} \Sigma P_{>m}\right)
$$

of 2.2 is an isomorphism of $\mathbb{Z} G$-modules.

PROOF. (1) is simply Proposition 2.2(1), with the observation that the homeomorphism is $G$-equivariant.

In (2) the $\mathbb{Z} G$-module structure of $\bigoplus_{m \in \mathscr{M}} \tilde{H}_{n-1}\left(P_{>m}\right)$ comes from the $\mathbb{Z} G$-module structure of $\bigoplus_{m \in \mathscr{N}} \tilde{C}_{n-1}\left(P_{>m}\right)$ in which $g\left(p_{0}<p_{1}<\cdots<p_{n-1}\right)=\left(g p_{0}<g p_{1}<\right.$ $\left.\cdots<g p_{n-1}\right)$ where $\left(p_{0}<p_{1}<\cdots<p_{n-1}\right)$ is an $(n-1)$-simplex of $P_{>m}$ and $\left(g p_{0}<g p_{1}<\cdots<g p_{n-1}\right)$ is an $(n-1)$-simplex of $P_{>g m}$. Thus $\bigoplus_{m \in \mathscr{M}} \tilde{H}_{n-1}\left(P_{>m}\right)$ is the direct sum of subspaces permuted by $G$ in which the stabilizer of each subspace is $G_{m}$, and the orbits of $G$ on these subspaces are in bijection with $G \backslash \mathscr{M}$. Thus with this action $\bigoplus_{m \in \mathscr{N}} \tilde{H}_{n-1}\left(P_{>m}\right)$ is an induced module as claimed. It is evident from the definition that $\mu$ is a homomorphism of $\mathbb{Z} G$-modules as claimed.

We now show the relevance of the wedge of suspensions construction in the situation where $P$ is an extension of $Q$ by minimal elements. As before we put $\mathscr{M}=P-Q$. Define

$$
j: \bigvee_{m \in \mathscr{A}} \Sigma P_{>m} \rightarrow P_{Q}
$$

as follows. Firstly $j(0)=0_{Q}$, secondly $j(m)=m$ for all $m \in \mathscr{M}$, and thirdly, for $x \in P_{>m} \times\{m\}$, we define $j(x)=j_{m}(x)$, where $j_{m}$ was defined before Proposition 2.2.

THEOREM 2.4. Suppose that $P$ is a $G$-poset which is an extension of $Q$ by minimal elements where $Q$ is $G$-invariant. Put $\mathscr{A}=P-Q$.

(1) $j: \bigvee_{m \in \mathscr{M}} \Sigma P_{>m} \rightarrow P_{Q}$ is a $G$-homotopy equivalence.

(2) For $n \geq 1$ the map

$$
\mu: \bigoplus_{m \in \mathscr{M}} \tilde{H}_{n-1}\left(P_{>m}\right) \rightarrow \tilde{H}_{n}\left(P_{Q}\right)
$$

is a $\mathbb{Z} G$-module isomorphism, where

$$
\mu\left(\sum_{m \in \mathscr{K}}\left[z_{m}\right]\right)=\sum_{m \in \mathscr{N}}\left[m * z_{m}-0_{Q} * z_{m}\right] .
$$

Here $z_{m} \in \tilde{Z}_{n-1}\left(P_{>m}\right)$ for all $m \in \mathscr{M}$. 
PrOOF. First note that $j$ is a $G$-equivariant map of posets. We show that for each $p \in P_{Q}, j^{-1}\left(\left(P_{Q}\right)_{\leq p}\right)$ is $G_{p}$-contractible. Then (1) follows from Theorem 1 in [6].

Case 1. There exists no $m \in \mathscr{M}$ with $m \leq p$.

In this case $j^{-1}\left(\left(P_{Q}\right)_{\leq p}\right)=\{0\}$ is evidently $G_{p}$-contractible.

Case 2. $p \in \mathscr{M}$.

In this case $j^{-1}\left(\left(P_{Q}\right)_{\leq p}\right)=\{p\}$ is also $G_{p}$-contractible.

Case 3. $p \notin \mathscr{M}$ and there exists $m \in \mathscr{M}$ such that $m<p$.

Let $\mathscr{M}_{p}=\{m \in \mathscr{M} \mid m<p\}$ and $X=\bigvee_{m \in \mathscr{M}} \Sigma P_{>m}$. Notice now that $j^{-1}\left(\left(P_{Q}\right)_{\leq p}\right)=\bigcup_{m \in \mathscr{M}_{p}} X_{\leq(p, m)}$ is a union of posets, each of which has a unique maximal member $(p, m)$ and which pairwise intersect in $\{0\}$. Such a poset is evidently contractible. For convenience, let $Y=j^{-1}\left(\left(P_{Q}\right)_{\leq p}\right)$ and $Z=\{(p, m) \mid m \in$ $\left.\mathscr{M}_{p}\right\} \cup\{0\} \subseteq X$. Then the two maps

$$
\begin{aligned}
& \psi: Y \rightarrow Z \\
& \phi: Z \rightarrow\{0\}
\end{aligned}
$$

defined by $\psi(0)=0$ and $\psi(y)=(p, m)$ if $0 \neq y \in X_{\leq(p, m)}$ and $\phi(p, m)=\phi(0)=0$ are $G_{p}$-equivariant and satisfy $y \leq \psi(y), z \geq \phi(z)$ always. Therefore by $[6,1.2] \psi$ and $\phi$ are $G_{p}$-homotopy equivalences which show that $Y$ is $G_{p}$-contractible.

Part (2) follows from (1) and Proposition 2.3.

We summarize the situation so far by combining Proposition 1.1 with Theorem 2.4 and a little bit more.

THEOREM 2.5. Suppose that $P$ is a G-poset which is an extension of $Q$ where $Q$ is $G$-invariant. Put $\mathscr{M}=P-Q$, and in (2) - (4) assume that $\mathscr{M}$ consists of minimal elements.

(1) There is a long exact sequence of $\mathbb{Z} G$-modules

$$
\cdots \rightarrow \tilde{H}_{n}(Q) \stackrel{\iota_{*}}{\longrightarrow} \tilde{H}_{n}(P) \stackrel{\kappa_{*}}{\longrightarrow} \tilde{H}_{n}\left(P_{Q}\right) \stackrel{r}{\longrightarrow} \tilde{H}_{n-1}(Q) \rightarrow \cdots
$$

where $\iota_{*}, \kappa_{*}$ are the maps on homology induced by the obvious inclusion maps $\iota, \kappa$.

(2) $P_{Q} \simeq_{G} \bigvee_{m \in \mathscr{M}} \Sigma P_{>m}$.

(3) For all $n \geq 0$

$$
\begin{aligned}
\tilde{H}_{n}\left(P_{Q}\right) & \cong \bigoplus_{m \in \mathscr{M}} \tilde{H}_{n-1}\left(P_{>m}\right) \\
& \cong \bigoplus_{m \in[G \backslash \mathscr{M}]} \tilde{H}_{n-1}\left(P_{>m}\right) \uparrow_{G_{m}}^{G}
\end{aligned}
$$

as $\mathbb{Z} G$-modules. 
(4) With respect to the first isomorphism of (3) the map $r$ of (1) is given by

$$
\bigoplus_{m \in \mathscr{M}}\left(\iota_{m}\right)_{*}
$$

where $\iota_{m}: P_{>m} \rightarrow Q$ is the inclusion map.

Proof. Part (1) is Proposition 1.1 and parts (2) and (3) come from Proposition 2.3 and Theorem 2.4. We now prove part (4). We must show that in the long exact sequence

$$
\cdots \rightarrow \tilde{H}_{n}(Q) \stackrel{\cdot}{\longrightarrow} \tilde{H}_{n}(P) \stackrel{\mu^{-1} \kappa_{*}}{\longrightarrow} \bigoplus_{m \in \mathscr{M}} \tilde{H}_{n-1}\left(P_{>m}\right) \stackrel{r \mu}{\longrightarrow} \tilde{H}_{n-1}(Q) \rightarrow \cdots
$$

the map $r \mu$ is given by

$$
r \mu=\bigoplus_{m \in \mathscr{M}}\left(\iota_{m}\right)_{*}
$$

where $\mu$ is the map of Theorem 2.4(2).

Let $h=\sum_{m \in \mathscr{M}}\left[z_{m}\right] \in \bigoplus_{m \in \mathscr{M}} \tilde{H}_{n-1}\left(P_{>m}\right)$. Then by Theorem 2.4(2), $\mu(h)=$ $\sum_{m \in \mathscr{M}}\left[m * z_{m}-0_{Q} * z_{m}\right]$. Notice now that $m * z_{m} \in P$ and $0_{Q} * z_{m} \in Q_{Q}$. Hence by Proposition 1.1(2) and Proposition 2.1(2), $r \mu(h)=\sum_{m \in \mathscr{M}} r\left(\left[m * z_{m}-0_{Q} * z_{m}\right]\right)=$ $\sum_{m \in \mathscr{M}}\left[\partial\left(m * z_{m}\right)\right]=\sum_{m \in \mathscr{M}}\left[z_{m}\right]$.

We conclude this section with a discussion of the multiple connectivity of the complexes $P$ and $P_{Q}$. For each simplicial complex $\Delta$ we denote by $\Delta^{(n)}$ the $n$ skeleton of $\Delta$.

LEMMA 2.6. Let $P$ be an extension of $Q$. If $Q$ is $n$-connected then the inclusion map $\iota: \Delta P^{(n)} \rightarrow \Delta P_{Q}^{(n)}$ is a homotopy equivalence, and $P$ is $n$-connected if and only if $P_{Q}$ is n-connected.

PROOF. For $p \in P, \iota^{-1}\left(\left(P_{Q}\right)_{\geq p}\right)=P_{\geq p}$ is contractible, while $\iota^{-1}\left(\left(P_{Q}\right)_{\geq 0_{Q}}\right)=Q$ is $n$-connected. Hence Lemma 4.3 in [2] completes the proof.

COROllary 2.7. Let $P$ be an extension of $Q$ by minimal elements and put $\mathscr{M}=$ $P-Q$. Assume that for all $m \in \mathscr{M}, P_{>m}$ is n-connected. Then

(1) $P_{Q}$ is $(n+1)$-connected.

(2) If $Q$ is $k$-connected, for $k \leq n+1$, then $P$ is $k$-connected.

PROOF. Since $P_{Q} \simeq \bigvee_{m \in \mathscr{M}} \Sigma P_{>m}$, and since the latter complex is $(n+1)$ connected, so is $P_{Q}$. Then (2) follows from Lemma 2.6 . 


\section{The proof of the Main Theorem}

We again work in the abstract setting of a poset $P$ which is an extension of $Q$, and suppose further that we are given a subset $I \subseteq Q$ such that $\mathrm{I}$ is an ideal in $P$. We will say that $I$ has the join property with respect to $Q$ (written in short $J Q$-property) if for each $q \in Q$ the set $I_{\leq q}$ is non-empty and has a join in $I$. Thus for each $q \in Q$ there is a unique largest member of $I$ less than or equal to $q$. We will say that $I$ has the strong join property with respect to $Q$ (written in short $S J Q$-property) if $I$ has the $J Q$-property and for all $m \in \mathscr{M}=P-Q$ and $i \in I$, whenever $m$ and $i$ have an upper bound in $P$ (and hence in $Q$ ) they have a join in $P$. In this case for each $m \in \mathscr{M}$ we denote $I_{\leq \geq m}=\{i \in I \mid\{m, i\}$ has an upper bound $\}$ regarded as a subposet of $I$. Note that in this definition and in Lemma 3.1 we do not require that the set $\mathscr{M}$ (defined to be $P-Q$ ) consist only of minimal elements.

Our application of these notions will be in the situation where $N \triangleleft G$ with $p|| N \mid$. We take $P=\mathscr{A}_{p}(G), Q=\left\{A \in \mathscr{A}_{p}(G) \mid A \cap N \neq 1\right\}$. Then $I=\mathscr{A}_{p}(N)$ has the $S J Q$-property.

LEMMA 3.1. Suppose that $P$ is a G-poset with $G$-invariant subsets $I \subseteq Q \subseteq P$ such that $P$ is an extension of $Q$ and $I$ is an ideal in $P$. Put $\mathscr{M}=P-Q$.

(1) If I has the JQ-property then the map $\phi: Q \rightarrow I$ defined by $\phi(q)=$ join of $I_{\leq q}$ is a $G$-homotopy equivalence with $G$-homotopy inverse the inclusion map $\iota: I \rightarrow Q$.

(2) If I has the SJQ-property then for all $m \in \mathscr{M}$ the map $\phi_{m}: Q_{>m} \rightarrow I_{\leq \geq m}$ defined by $\phi_{m}(q)=\phi(q)$ is a $G_{m}$-homotopy equivalence with $G_{m}$-homotopy inverse $\psi_{m}: I_{\leq \geq m} \rightarrow Q_{>m}$ defined by $\psi_{m}(i)=$ join of $\{m, i\}$.

PROOF. All of the maps mentioned are equivariant maps of posets, and they satisfy

$$
\begin{aligned}
\phi \iota & =\mathrm{id}_{I}, \\
\iota \phi(q) & \leq q \text { for all } q \in Q, \\
\psi_{m} \phi_{m}(q) & \leq q \text { for all } q \in Q_{>m}, \\
\phi_{m} \psi_{m}(i) & \geq i \text { for all } i \in I_{\leq \geq m} .
\end{aligned}
$$

Hence by $[6,1.1] \iota, \phi$ and $\psi_{m}, \phi_{m}$ are pairs of mutually inverse equivariant homotopy equivalences.

We will apply Lemma 3.1 in the situation where $P$ is an extension of $Q$ by minimal elements, in which case $P_{>m}=Q_{>m}$.

THEOREM 3.2. Suppose that $P$ is a G-poset which is an extension of $Q$ where $Q$ is $G$-invariant. Put $\mathscr{M}=P-Q$, and in (2) - (4) assume that $\mathscr{M}$ consists of minimal elements. Suppose that $I \subseteq Q$ is a $G$-invariant ideal of $P$ with the $S J Q$-property. 
(1) There is a long exact sequence of $\mathbb{Z} G$-modules

$$
\cdots \rightarrow \tilde{H}_{n}(I) \stackrel{\iota \cdot}{\longrightarrow} \tilde{H}_{n}(P) \stackrel{\kappa_{\bullet}}{\longrightarrow} \tilde{H}_{n}\left(P_{Q}\right) \stackrel{s}{\longrightarrow} \tilde{H}_{n-1}(I) \rightarrow \cdots
$$

where $\iota_{*}, \kappa_{*}$ are the maps on homology induced by the obvious inclusion maps $\iota, \kappa$.

(2) $P_{Q} \simeq_{G} \bigvee_{m \in \mathscr{M}} \Sigma I_{\leq \geq m}$.

(3) For all $n \geq 0$

$$
\begin{aligned}
\tilde{H}_{n}\left(P_{Q}\right) & \cong \bigoplus_{m \in \mathscr{M}} \tilde{H}_{n-1}\left(I_{\leq \geq m}\right) \\
& \cong \bigoplus_{m \in[G \backslash \mathscr{M}]} \tilde{H}_{n-1}\left(I_{\leq \geq m}\right) \uparrow_{G_{m}}^{G}
\end{aligned}
$$

as $\mathbb{Z} G$-modules.

(4) With respect to the first isomorphism of (3) the map $s$ of (1) is given by

$$
\bigoplus_{m \in \mathscr{M}}\left(\iota_{m}\right)_{*}
$$

where $\iota_{m}: I_{\leq \geq m} \rightarrow I$ is the inclusion map.

PROOF. (1) We identify the terms in the long exact sequence of Theorem 2.5 using the homology isomorphisms which we deduce from Lemma 3.1. It may help to consider the following diagram in which the top row is the sequence of Theorem 2.5 and all vertical arrows are isomorphsms, $\mu$ having been defined in Theorem 2.4 .

$$
\begin{aligned}
& \cdots \quad \longrightarrow \quad \tilde{H}_{n}(Q) \stackrel{\iota}{\longrightarrow} \tilde{H}_{n}(P) \stackrel{\kappa_{*}}{\longrightarrow} \quad \tilde{H}_{n}\left(P_{Q}\right) \quad \stackrel{r}{\longrightarrow} \tilde{H}_{n-1}(Q) \\
& \bigoplus \tilde{H}_{n-1}^{\mu}\left(P_{>m}\right) \quad \tilde{\phi}_{*} \downarrow \overbrace{}^{\ell *} \\
& \oplus \phi_{m *} \downarrow \uparrow \oplus \psi_{m *} \\
& \bigoplus \tilde{H}_{n-1}\left(I_{\leq \geq m}\right)
\end{aligned}
$$

The sequence presented here thus has $s=\phi_{*} r$.

(2) The homotopy equivalences $\psi_{m}$ yield a $G$-homotopy equivalence

$$
\vee \Sigma \psi_{m}: \bigvee_{m \in \mathscr{M}} \Sigma I_{\leq \geq m} \rightarrow \bigvee_{m \in \mathscr{M}} \Sigma P_{>m},
$$

noting here that $P_{>m}=Q_{>m}$. Combining this with Theorem 2.5(2) gives the result.

(3) The first isomorphism is the composite

$$
\bigoplus_{m \in \mathscr{M}} \tilde{H}_{n-1}\left(I_{\leq \geq m}\right) \stackrel{\oplus \psi_{m}}{\longrightarrow} \bigoplus_{m \in \mathscr{M}} \tilde{H}_{n-1}\left(P_{>m}\right) \stackrel{\mu}{\longrightarrow} \tilde{H}_{n}\left(P_{Q}\right),
$$


where in Theorem 2.4 we saw that $\mu$ is an isomorphism. The argument that this is an induced module was given in Proposition 2.3(2).

(4) On considering the diagram presented in the proof of (1) we see that we have to show $\phi_{*} r \mu \psi_{m *}=\iota_{m *}$ where $\iota_{m}: I_{\leq \geq m} \rightarrow I$. We saw in Theorem 2.5(4) that $r \mu=\oplus \iota_{m *}$, where in that case $\iota_{m}: P_{>m} \rightarrow Q$. Since $\phi_{m}$ is the restriction of $\phi$ to $P_{>m}$ we have $\iota_{m} \phi_{m}=\phi \iota_{m}$ and so

$$
\begin{aligned}
\phi_{*} r \mu \psi_{m *} & =\phi_{*}\left(\oplus \iota_{m *}\right) \psi_{m *} \\
& =\phi_{*} \iota_{m *} \psi_{m *} \\
& =\iota_{m *} \phi_{m *} \psi_{m *} \\
& =\iota_{m *}
\end{aligned}
$$

as required, since $\phi_{m}$ and $\psi_{m}$ are mutually inverse homotopy equivalences.

We now translate Theorem 3.2 into the group theoretic situation of the Main Theorem. We first verify separately one of the identifications that will be needed for this.

LEMMA 3.3. Let $G$ be a finite group, $p$ a prime and $N \triangleleft G$. Then

$$
\mathscr{A}_{p}(N)_{\leq \geq A}=\mathscr{A}_{p}\left(C_{N}(A)\right) \text {. }
$$

PROOF. We have

$$
\mathscr{A}_{p}(N)_{\leq \geq A}=\left\{K \in \mathscr{A}_{p}(N) \mid A \text { and } K \text { have an upper bound }\right\} .
$$

Now $A$ and $K$ have an upper bound precisely if they are subgroups of some elementary abelian subgroup, which happens precisely if $A$ and $K$ centralize each other, that is $K \leq C_{N}(A)$.

ProOF OF THE MAIN THEOREM. Let $G, p$ and $N$ be as in the main theorem. Let $P=\mathscr{A}_{p}(G), I=\mathscr{A}_{p}(N)$ and $Q=\{A \in P \mid A \cap N \neq 1\}$. Notice that $P_{Q}=$ $\mathscr{A}_{p}(G)_{N}$. It is almost immediate that $P, Q$ and $I$ satisfy all the hypotheses of Theorem 3.2, and of course $P$ is a $G$-poset under conjugation and $Q, I$ are $G$ invariant. The Main Theorem is the statement of Theorem 3.2 in this particular case.

We check two things in particular. If $A \in P-Q$ then $A$ is an elementary abelian $p$-group with $A \cap N=1$, so by hypothesis $A$ has order $p$. Thus $P$ is an extension of $Q$ by minimal elements and $P-Q=\mathscr{M}$ as defined in the statement of the Main Theorem. Secondly, for $A \in \mathscr{M}$ we have $I_{\leq \geq A}=\mathscr{A}_{p}\left(C_{N}(A)\right)$ by Lemma 3.3. 


\section{The Lefschetz invariant and the generalized Steinberg module}

As far as computing the homology of $\mathscr{A}_{p}(G)$ is concerned, we were able in our Main Theorem to give a sharp description of $\mathscr{A}_{p}(G)_{N}$ only in the case when all elementary abelian $p$-subgroups $A \leq G$ satisfying $A \cap N=1$ are cyclic. If we weaken the type of information we try to compute and do not ask for the full homology, we may give further results without the strong hypothesis on $N$.

Following [5], if $\Delta$ is a $G$-simplicial complex we shall use the notation

$$
\begin{aligned}
\tilde{\Lambda}(\Delta) & =\sum_{i=-1}^{\operatorname{dim} \Delta}(-1)^{i} \Delta_{i}, \\
\tilde{L}(\Delta) & =\sum_{i=-1}^{\operatorname{dim} \Delta}(-1)^{i} C_{i}(\Delta)
\end{aligned}
$$

respectively for the reduced Lefschetz invariant of $\Delta$ in the Burnside ring of $G$, and the reduced Lefschetz module of $\Delta$ in the Green ring of $R G$-modules. Here $\Delta_{i}$ is the $G$-set of $i$-simplices of $\Delta$, and $C_{i}(\Delta)$ is the $i$-dimensional chain group of $\Delta$, taken over a commutative coefficient ring $R$. In order to make sense of the Green ring of finitely generated $R G$-modules we will suppose that $R$ is either a field or a complete discrete valuation ring. We take $\Delta_{-1}$ to be a single point with trivial $G$-action, and $C_{-1}(\Delta)=R$ the trivial module. As usual, in case $\Delta=\Delta(P)$ arises from a poset $P$, we will write simply $\tilde{\Lambda}(P)$ and $\tilde{L}(P)$.

The generalized Steinberg module of the finite group $G$ at the prime $p$ is defined to be

$$
S t_{p}(G)=\tilde{L}\left(\mathscr{A}_{p}(G)\right)
$$

as an element of the Green ring of $R G$-modules. A survey of some of its properties can be found in [7]. There is a homomorphism from the Burnside ring to the Green ring which takes every $G$-set to the corresponding permutation module, and evidently $S t_{p}(G)$ is the image of $\tilde{\Lambda}\left(\mathscr{A}_{p}(G)\right)$ under this homomorphism. We will prove our identities for $S t_{p}(G)$ by first establishing the corresponding identities for $\tilde{\Lambda}\left(\mathscr{A}_{p}(G)\right)$ and then applying this homomorphism.

We are about to invoke a theorem of Thévenaz which applies to posets of the form $A+{ }_{C} B$ where $A$ and $B$ are posets and $C$ is an ideal in the poset $A \times B$. The poset $A+{ }_{C} B$ is defined to be the poset whose underlying set is the disjoint union of $A$ and $B$, and whose ordering is defined by the ordering of $A$, the opposite ordering of $B$ and the extra condition:

$$
\text { if } a \in A, b \in B \text { and }(a, b) \in C \text { then } a<b .
$$

If $A$ and $B$ are $G$-posets and if $C$ is a $G$-invariant ideal then $A+{ }_{C} B$ is again a $G$-poset. 
In such a poset, $A$ is an ideal of $A+{ }_{C} B$, and $B$ is an ideal of the opposite poset. Conversely, in every poset $P$ with a subset $B$ which is an ideal in $P^{\text {op }}$, the complementary set $A=P-B$ is an ideal in $P$, and $P=A+{ }_{c} B^{\text {op }}$ where $C=\{(x, y) \mid x \in A, y \in B, x<y\}$. Thus Thévenaz's theorem applies to our situation where $P$ is an extension of $Q$ and $\mathscr{M}=P-Q$, so that $P=\mathscr{M}+{ }_{C} Q^{\text {op }}$.

THEOREM 4.1. Suppose that $P$ is a G-poset which is an extension of $Q$ where $Q$ is $G$-invariant. Put $\mathscr{M}=P-Q$.

(1)

$$
\tilde{\Lambda}(P)=\tilde{\Lambda}(Q)+\sum_{m \in[G \backslash \mathscr{M}]}\left(\tilde{\Lambda}\left(\mathscr{K}_{<m}\right) \cdot \tilde{\Lambda}\left(Q_{>m}\right)\right) \uparrow_{G_{m}}^{G} .
$$

(2) Suppose further that I is a $G$-invariant ideal of $P$ with $I \subseteq Q$, and that I has the SJQ-property. Then

$$
\tilde{\Lambda}(P)=\tilde{\Lambda}(I)+\sum_{m \in[G \backslash \mathscr{M}]}\left(\tilde{\Lambda}\left(\mathscr{M}_{<m}\right) \cdot \tilde{\Lambda}\left(I_{\leq \geq m}\right)\right) \uparrow_{G_{m}}^{G} .
$$

PROOF. Part (1) is a restatement of the first formula of $[5,3.3]$ in the present context. Part (2) follows from part (1) and Lemma 3.1, since $\tilde{\Lambda}$ is constant on $G$-homotopy equivalent complexes $[5,1.3]$.

THEOREM 4.2. Suppose that $G$ is a finite group with a normal subgroup $N$ such that $p|| N \mid$. Then

$$
\tilde{\Lambda}\left(\mathscr{A}_{p}(G)\right)=\tilde{\Lambda}\left(\mathscr{A}_{p}(N)\right)+\sum_{\substack{A \in \mathscr{A}_{p}(G), A \cap N=1 \\ \text { up to conjugacy }}}\left(\tilde{\Lambda}\left(\mathscr{A}_{p}(G L(A))\right) \cdot \tilde{\Lambda}\left(\mathscr{A}_{p}\left(C_{N}(A)\right)\right)\right) \uparrow_{N_{G}(A)}^{G}
$$

in the Burnside ring, and so

$$
S t_{p}(G)=S t_{p}(N)+\sum_{\substack{A \in \mathscr{A}_{p}(G), A \cap N=1 \\ \text { up to conjugacy }}}\left(S t_{p}(G L(A)) \otimes S t_{p}\left(C_{N}(A)\right)\right) \uparrow_{N_{G}(A)}^{G}
$$

in the Green ring.

PROOF. As in the proof of the Main Theorem in Section 3 we take $P=\mathscr{A}_{p}(G)$, $I=\mathscr{A}_{p}(N), Q=\{A \in P \mid A \cap N \neq 1\}$ and $\mathscr{M}=\{A \in P \mid A \cap N=1\}$. We have seen in Lemma 3.3 that $I_{\leq \geq A}=\mathscr{A}_{p}\left(C_{N}(A)\right)$ in this situation, and it is clear that $\mathscr{M}_{<A}$ is the poset of proper subspaces of $A$. This is in fact the poset which defines the building of $G L(A)$, and so we write $S t_{p}(G L(A))$ for its Lefschetz module in the final equation. 
The most straightforward (non-trivial) situation in which we may apply the formula for the Steinberg module in Theorem 4.2 occurs when $G$ satisfies the condition that $A \cap N=1, A$ an elementary abelian subgroup of $G$ implies $A$ is cyclic. In this case, if $A \cong C_{p}$ then $A$ has no proper subspaces and so $S t_{p}(G L(A))=-R$. Thus

$$
S t_{p}(G)=S t_{p}(N)-\sum_{\substack{A \in \mathcal{A}_{p}(G), A \cap N=1 \\ \text { up to conjugacy }}} S t_{p}\left(C_{N}(A)\right) \uparrow_{N_{G}(A)}^{G} .
$$

This formula may also be deduced by taking the alternating sum of the terms in the long exact sequence of the Main Theorem. It seems that this formula is known to Bouc [3] who uses it to relate $S t_{2}\left(S_{n}\right)$ and $S t_{2}\left(A_{n}\right)$.

\section{Examples}

We present two examples to illustrate the theory we have developed. In the first we consider the situation $A_{n} \triangleleft S_{n}$, and only state the result when $n \geq 6$ to avoid the exceptional small cases which may easily be worked by these and other methods.

PROPOSITION 5.1. For each $n \geq 6$ there is a long exact sequence

$$
\begin{aligned}
& \cdots \quad \longrightarrow \quad \tilde{H}_{s}\left(\mathscr{A}_{2} A_{n}\right) \quad \longrightarrow \quad \tilde{H}_{s}\left(\mathscr{A}_{2} S_{n}\right) \quad \longrightarrow \quad \tilde{H}_{s-1}\left(\mathscr{A}_{2} S_{n-2}\right) \uparrow_{S_{2} \times S_{n-2}} \\
& \longrightarrow \tilde{H}_{s-1}\left(\mathscr{A}_{2} A_{n}\right) \longrightarrow \tilde{H}_{s-1}\left(\mathscr{A}_{2} S_{n}\right) \longrightarrow \tilde{H}_{s-2}\left(\mathscr{A}_{2} S_{n-2}\right) \uparrow_{S_{2} \times S_{n-2}} \\
& \cdots \quad \longrightarrow \quad \tilde{H}_{1}\left(\dot{\mathscr{A}}_{2} A_{n}\right) \quad \longrightarrow \quad \tilde{H}_{1}\left(\dot{\mathscr{A}}_{2} S_{n}\right) \quad \longrightarrow \quad 0 .
\end{aligned}
$$

PROOF. We apply the Main Theorem, in which the conjugacy classes of the set $\mathscr{M}$ are represented by the groups $\langle(1,2)\rangle,\langle(1,2)(3,4)(5,6)\rangle,\langle(1,2)(3,4)(5,6)$ $(7,8)(9,10)\rangle, \ldots$ Let us write $t_{r}=(1,2)(3,4) \cdots(4 r+1,4 r+2) \in S_{n}$. Then $C_{A_{n}}\left(t_{r}\right)$ contains the subgroup of index 2 in $\langle(1,2),(3,4), \ldots,(4 r+1,4 r+2)\rangle$ which consists of even permutations, and this is a normal 2-subgroup of $C_{A_{n}}\left(t_{r}\right)$. Thus $\mathscr{A}_{2}\left(C_{A_{n}}\left(t_{r}\right)\right)$ is contractible, except when $r=0$, in which case $C_{A_{n}}(1,2) \cong S_{n-2}$. This gives the general terms of the long exact sequence, and it terminates as indicated because all of the spaces $\mathscr{A}_{2}\left(C_{A_{n}}\left(t_{r}\right)\right)$ are connected.

For our second example we consider a Frobenius group $C_{q} \rtimes C_{p}$ where $p$ is prime with $p \mid q-1$, and form the wreath product $G=\left(C_{q} \rtimes C_{p}\right)$ i $C_{p}$. The base group $N=\left(C_{q} \rtimes C_{p}\right)^{p}$ is a normal subgroup of index $p$. This example is of interest because of counterexamples provided by Alperin to an incorrect conjecture made earlier by one of us $[7,3.2]$. The conjecture was that $\tilde{H}_{n}\left(\mathscr{A}_{p}(G)\right) \otimes \mathbb{Z}_{p}$ should always be a projective $\mathbb{Z}_{p} G$-module, where $\mathbb{Z}_{p}$ denotes the $p$-adic integers, but in [1] Alperin shows that the 
top non-vanishing homology group of $\mathscr{A}_{p}\left(S_{p^{2}}\right)$ with $p \geq 5$, is not projective. He has also observed that the wreath product group $G$ provides a counterexample if $p \geq 3$, using a similar argument to the one of $S_{p^{2}}$. We will now compute the homology of $\mathscr{A}_{p}(G)$.

PROPOSITION 5.2. Let $G=\left(C_{q} \rtimes C_{p}\right)$ ᄀ $C_{p}$ where $C_{q} \rtimes C_{p}$ is a Frobenius group, $p$ a prime, and let $N=\left(C_{q} \rtimes C_{p}\right)^{p}$ be the base group. Put $V=\tilde{H}_{0}\left(\mathscr{A}_{p}\left(C_{q} \rtimes C_{p}\right)\right)$, the coordinate-sum zero submodule of rank $q-1$ in the permutation module on the $q$ Sylow $p$-subgroups of $C_{q} \rtimes C_{p}$. If $p \neq 2$ then

$$
\tilde{H}_{n}\left(\mathscr{A}_{p}(G)\right)= \begin{cases}V \uparrow_{N}^{\otimes G} & \text { if } n=p-1, \\ V \uparrow_{\delta\left(C_{q} \rtimes C_{p}\right) \times C_{p}} & \text { if } n=1, \\ 0 & \text { otherwise. }\end{cases}
$$

If $p=2$ then $\tilde{H}_{n}\left(\mathscr{A}_{p}(G)\right)=0$ unless $n=1$, in which case there is a short exact sequence

$$
0 \rightarrow V \uparrow_{N}^{\otimes G} \rightarrow \tilde{H}_{1}\left(\mathscr{A}_{p}(G)\right) \rightarrow V \uparrow_{\delta\left(C_{q} \rtimes C_{p}\right) \times C_{p}}^{G} \rightarrow 0 .
$$

To explain the notation, we may regard $V$ as a module for $N=\left(C_{q} \rtimes C_{p}\right)^{p}$ via projection onto the first $C_{q} \rtimes C_{p}$ factor. Then $V \uparrow \uparrow_{N}^{\otimes G}$ denotes the tensor induction. We consider also the diagonal subgroup $\delta\left(C_{q} \rtimes C_{p}\right) \subseteq N$, and regard $V$ as a module for $\delta\left(C_{q} \rtimes C_{p}\right) \times C_{p}$, where the $C_{p}$ factor performs the wreath action, via projection onto $\delta\left(C_{q} \rtimes C_{p}\right) \cong C_{q} \rtimes C_{p}$.

PROOF. One sees easily that all complements to $N$ in $G$ are conjugate, since any two are conjugate to complements for the base group in $C_{p} i C_{p} \cong \mathbb{F}_{p} C_{p} \rtimes C_{p}$ and here complements are conjugate since $H^{1}\left(C_{p}, \mathbb{F}_{p} C_{p}\right)=0$. The centralizer in $N$ of such a complement is $\delta\left(C_{q} \rtimes C_{p}\right)$ and so the long exact sequence of the Main Theorem takes the form

$$
\cdots \rightarrow \tilde{H}_{n}\left(\mathscr{A}_{p}(N)\right) \rightarrow \tilde{H}_{n}\left(\mathscr{A}_{p}(G)\right) \rightarrow \tilde{H}_{n-1}\left(\mathscr{A}_{p}\left(C_{q} \rtimes C_{p}\right)\right) \uparrow_{\delta\left(C_{q} \rtimes C_{p}\right) \times C_{p}}^{G} \rightarrow \cdots
$$

Now $\mathscr{A}_{p}\left(C_{q} \rtimes C_{p}\right)$ is the set of $q$ Sylow $p$-subgroups of $C_{q} \rtimes C_{p}$ and so its homology is zero except in dimension zero, where it is $V$. As for $\mathscr{A}_{p}(N)$, this is homotopy equivalent to the join $\mathscr{A}_{p}\left(C_{q} \rtimes C_{p}\right) * \cdots * \mathscr{A}_{p}\left(C_{q} \rtimes C_{p}\right)$ with $p$ factors by [4], and using the methods of [6] it is easy to see that Quillen's homotopy equivalence is equivariant for the action of $G$ with $C_{p}$ permuting the factors. Since $\mathscr{A}_{p}\left(C_{q} \rtimes C_{p}\right)$ is just a set of $q$ points, or in other words a wedge of $(q-1) 0$-spheres, the join of $p$ copies of this space is a wedge of $p(q-1)(p-1)$-spheres, in which each $C_{q} \rtimes C_{p}$ factor of $N$ permutes the corresponding set of $(q-1)$ of these spheres. Thus we have

$$
\tilde{H}_{n}\left(\mathscr{A}_{p}(N)\right)= \begin{cases}V \otimes \cdots \otimes V & \text { if } n=p-1 \\ 0 & \text { otherwise. }\end{cases}
$$


In the action on this homology the wreathing $C_{p}$ permutes the factors $V$ in the tensor product and so

$$
\tilde{H}_{p-1}\left(\mathscr{A}_{p}(N)\right)=V \uparrow_{N}^{\otimes G} .
$$

Substituting these identifications into the long exact sequence completes the proof of this result.

COROLlaRY 5.3. (Alperin). Let $G$ be the group specified in Proposition 5.2. When $p \geq 3, H_{p-1}\left(\mathscr{A}_{p}(G)\right) \otimes \mathbb{Z}_{p}$ is not a projective $\mathbb{Z}_{p} G$-module.

PROOF. As a module for the wreathing $C_{p}$, the tensor induced module $V \uparrow \stackrel{\otimes G}{N}$ is a permutation module on tensors $v_{i_{1}} \otimes \cdots \otimes v_{i_{p}}$, where $v_{1}, \ldots, v_{q-1}$ is some basis of $V$. It has as a direct summand the trivial module, spanned by $v_{1} \otimes \cdots \otimes v_{1}$, which is not projective.

\section{Acknowledgement}

The authors are grateful to the Department of Mathematics at the California Institute of Technology for the hospitality afforded them. In the case of Y. Segev this extended to sabbatical support during the writing of this paper.

\section{References}

[1] J. L. Alperin, 'A Lie approach to finite groups', in: Groups-Canberra 1989 (ed. L. G. Kovács), Lecture Notes in Math. 1456 (Springer, Berlin, 1990) pp. 1-9.

[2] M. Aschbacher and Y. Segev, 'Locally connected simplicial maps', Israel J. Math. 77 (1992), 285-303.

[3] S. Bouc, 'Exponentielle et modules de Steinberg', preprint.

[4] D. Quillen, 'Homotopy properties of the poset of nontrivial $p$-subgroups of a group', Adv. in Math. 28 (1978), 101-128.

[5] J. Thévenaz, 'Permutation representations arising from simplicial complexes', J. Combin. Theory Ser. A 46 (1987), 121-155.

[6] J. Thévenaz and P. J. Webb, 'Homotopy equivalence of posets with a group action', J. Combin. Theory Ser. A 56 (1991), 173-181.

[7] P. J. Webb, 'Subgroup complexes', in: The Arcata conference on representations of finite groups (ed. P. Fong), Proceedings of Symposia in Pure Mathematics vol. 47 (American Mathematical Society, Providence, R. I., 1987) pp. 349-365.

Department of Mathematics

Ben - Gurion University

Beer - Sheva 84105

Israel
Department of Mathematics

University of Minnesota

Minneapolis

Minnesota 55455, USA 